\title{
Increasing Quality and Access to Oral Health Care through Public-Private Partnerships
}

\author{
Mosha HJ. \\ Consultant Dental Surgeon. Mosha Medical and Dental Clinic Dar es Salaam
}

Mosha HJ: Increasing Quality and Access to Oral Health Care through Public-Private Partnerships. Tanz Debt J 2008; 15 (1): $27-32$

\begin{abstract}
Partneships signifies a legal relationship between two or more entities contractually associated as joint principles in a business. Public Pivate Partneship for health (PPPH), involves two or more entities from the public and private sectors that engage in a joint endeavour to achieve common health goals and objectives. The goal for the PPPH is to contribute to the strengthening of the National Health System with the capabilities and full participation of the Private Health Sector to maximize attainment of the national health goals. This paper looks at how quality and access to OHC through PPPH, can be attained by contracting private dentists to provide OHCS in public health facilities. Contracted dentists refer to private dentists who provide dental services, on behalf of the health facility (HF), to health facility patients, either in the dentists' private clinic or in a dedicated space located within the health facility. When contracting, dentists and health facility may elect to contract based on the specific services provided; number of patients to be seen; number of visits available to HF patients; number of sessions to be committed to HF patients; or any other mutually agreeable basis.

Currently private practitioners are regarded as partners in the provision of $\mathrm{OHC}$ services rather than competitors and the role of the government is to provide a conducive environment for establishing private practice. By contracting private dentists, it can increase access and quality of OHCS provided to underserved groups in the rural health facilities in a manner that is consistent with applicable health facility policies and procedures. Public Private Partneships provides opportunity for looking at health in a comprehensive way. It is built on the strength of organizations allowing them to pool resources, avoid duplication and minimize impact. It encourages the involvement of communities in their health care and focus attention on neglected health problems, attracts new resources and use them efficiently and fill service gaps and increase access to underserved groups. Therefore increasing access to quality $\mathrm{OHC}$ services in the rural areas can be attained through contracting private dentists to provide $\mathrm{OHC}$ services in $\mathrm{PHC}$ facilities in these areas.
\end{abstract}

Correspondance: Mosha HJ, P.O. Box 40384 Dar es Salaam, Tanzania. E-mail moshahj@yahoo.com

\section{Introducttion}

Oral health is an inseparable part of total health but has often been treated as if it were not and left to develop rather independently. This attitude has led to the common experience of initial neglet of oral health sector because a low priority is allocated to it. This is followed by inordinate expenditure, private or public, on curative, restorative and rehabilitative services demanded by a public. This has allowed the prevalence of oral diseases to progress from low to high. Yet the main oral diseases are eminently preventable or controlable. Mainly through the use of fluorides, developed countries are experiencing a return to the low levels of dental caries that once existed and are maintaining an improved regime to control periodontal disease (1).

In developing contries the changing enviroment and lifestyle which used to be consistent with extremely low levels of dental caries now calls for carefully coordinated delivery systems focused on use of available effective preventive methods. The philosophy and goals in oral health care are, to concentrate on a preventive approch to optimization of the relevant factors in the environment and lifestyle, using methodology which we have now or can soon develop, and in doing so achieve a massive social improvement in health and economic benefit. The results of the International Collaborative Study on Oral Health Care Systems stressed that, oral health is inseparable from health. In developing oral health programmes, priority should be given to preventive approaches, optimizing those factors in the environment and lifestyle which can bring about decisive improvements in people's health and quality of life. In which case the practical, action oriented approaches used to improve the management of oral health services, could be adopted to various health, social and economic environments and should be used in all communities (2). Dentistry is an established independent profession with government regulation. With the development of academic training, the former tooth drawers, barbers and unlicenced dental therapists came to be replaced by licenced trained practitioners (3). Oral health care, like many other social and health developments expanded rapidily in the context of industrilization, conciding with large increase in caries rates in industrializing nations. To concentrate on the increase in dental diseases, oral 
health care professionals began actively promoting oral hygiene and the use of oral health care services $(4,5)$. This was followed by the commercial development and promotion of oral health care products, such as toothbrushes and toothpaste. This process of modernization influenced people's oral hygiene behavour. More manufacturers began to promote toothbrushes and pastes and oral practioners began to provide education on the benefits of oral hygiene. Mass communication improved and people become more concerned with individual health and ways to preserve it (6). Solving health and social problems requires collaboration among organisations from different sectors and at different levels. Inter-sectoral approch to health services delivery is more efficient and cost-effective than separate uncoordinated efforts. Political and economic changes, which have created opportunities for the private and public sectors to partner, include Health Sector Reforms and increasing pressures on resources for health.

\section{Public-Private Partnership in Health (PPPH)}

Partnership signifies a legal relationship between two or more entities contractually associated as joint principles in a business. Usually each of these entities has specific rights and responsibilities related to their partnership. Partners tend to pool their resources, either technical, organisational, geographical, human or financial. The goal for the Public Private Partnership in Health is to contribute to strengthening the National Health System with the capabilities and full participation of the Private Health Sector to maximize attainment of the national health goals. The main objective of partnership is to establish a functional integration and a sustained operation of a pluralistic health care delivery system by optimizing the equitable use of the available resources and investing in comparative advantages of the partners.

PPP in health involves two or more entities from the public and private sectors that engage in a joint endeavour to achieve common health goals and objectives. Partnerships can be informal or formal (in a form of written agreement or a contract). In establishing a partnership, potential partners must consider and discuss the goal of the partnership, type of agreement to guide the partnership, time frame of the partnership, recourses to be committed for the partnership, target population to be reached and roles and responsibilities for each partner. Public Private Partneships provides opportunity for looking at health in a comprehensive way. It builds on the strengths of each organisation, allowing them to pool resources, avoid duplication and minimize impact. PPPH also encourege the involvement of communities in their health care and focus attention on neglected health problems. Also it attracts new resources and use them efficiently, fill service gaps and increase access to services for underserved groups (7).
The areas of partnership include; policy formulation and development, co-ordination and planning, financial resource mobilization and allocation, human resource for health management, monitoring and evaluation of health care delivery. The Key factors in an effective partnership include communication (talking to each other about the partneship), cooperation (reaching an understanding to assist each other), coordination (combining resources and strengthening individual roles) and collaboration (working together to develop plans, review policies or formulate new ones). The guiding principles of the partnership are that the scope and level of the partnership depends on the extent to which partners' missions coincide. The overall responsibility for health policy formulation and for the health status of the population is maintained by Central Government which will consult and aim for consensus with the partners in all cases of common concern. Provision of health services to the population pertains to the different partners (Local Government and private sector), guided by the decentralisation policy. Within a given context partners strive to complement their services rather than duplicating them. The identity and autonomy of each partner is respected. Equitable allocation of resources for health and is in accordance with the volumes and quality of the contribution to the implementation of the HSSP and inputs, outputs and outcomes pertaining to attainment of the public health goals are agreed, reported by and shared among the partners.

\section{Main sub-sectors of the private sector in health}

Private Not for Profit (PNFP) providers are motivated by concern for the welfare of the population. The PNFP comprise of agencies that provide health services from an established static health unit/facility to the population and those that work with communities and other counterparts to provide non facility-based health services. The Non-facility based PNFP providers comprise of the majority of local and international organisations working in the health sector commonly referred to as NGOs. They work with counterparts such as government, facility-based PNFP providers, private practitioners and communities. Their contribution is in areas ranging from social awareness and advocacy to more specific aspects of service delivery. The area of emphasis tends to conform to agency expertise such as special disease programmes, technical assistance, training, capacity building, emergency and relief services and mainstream service delivery. The facility-based has a large infrastructure base comprising of a network of hospitals and health centres and lower level units in the country. They provide health services and train health workers. The Private Health Practitioners (PHP) encompasses all cadres of 
health professionals in the Clinical, Dental, Diagnostics, Medical, Midwifery, Nursing, Pharmacy and Public Health categories who provide private health services outside the Public, PNFP and the Traditional and Complementary Medicine establishment. The Private health practitioners provide mainly primary level services and limited secondary level services. A few urban units offer tertiary and specialist care. Traditional and Complementary Medicine Practitioners include all types of traditional healers: i.e. Herbalists, Spiritual healers, Bone Setters, Traditional Birth Attendants, Hydro Therapists, Traditional Dentists, etc. It does not include people who engage in harmful practices, casting of spells and child sacrifice (9).

\section{Rationale of the Partnership}

Reform of the Health Sector seeks to improve equity, access, efficiency, quality and sustainability of health care. A partnership with the private health sector accelerates the attainment of these objectives. Equity is cross cutting and involves issues of access, quality, and financing especially for the most vulnerable groups of the population. Subsiding private provision of the Minimum Health Care Package will increase the proportion of the population that can have access. Encouraging higher income segments of the population to use unsubsidised private providers can allow for more public funds to be used to provide priority services to those who cannot afford to pay. Private health providers may offer services in areas where there are no existing public health facilities and may be available during more convenient hours. Efficiency involves rationalising health inputs to ensure maximum output. The private health sector is able to contribute to health inputs and since these are taken into account within the partnership, represent a cost minimisation to the public sector. A partnership to improve efficiency, considers complementarity's and minimises duplication of services where possible. By pro viding the population with a choice of health provider, the providers will strive to offer quality services. Minimum standards are agreed within the partnership and self-regulation is encouraged. Private providers can contribute to sustainability by creating an open market for health services, not restricted by changes in the government policies and budgetary constraints. The mixed system (Public-Private Health system) thus created would be stronger and can compensate for weaknesses in either provider. The private Health Infrastructure represents a national asset that needs to be preserved.

\section{Increasing Access to Oral Health Care through Public-Private Partnerships in Health}

Primary health services include services provided by a licensed dentist or other qualified personnel. A health facility patient is someone who uses the services of the health facility as his or her principal source of primary health care. A patient typically resides in the HF's service area and is able to reasonably access primary and preventive care services at the Health Facility. The term dentist and contracted dentists refer exclusively to private dentists who provide dental services on behalf of the HF, to HF patients, either in the dentist's private practice or in a dedicated space located within the HFs.

There are currently in Tanzania 250 trained oral health workers and 114 support staff providing oral health services. They are located in only 87 out of a total of 121 districts. With the initiation of the provision of urgent dental treatment at the rural health centres and strategically located dispensaries 255 service units have been established and are manned by Clinical Officers (COs) trained to provide basic dental treatment. The provision of health services in Tanzania is guided by the National Health Policy (11) which aims at providing direction towards improvement and sustainability of the health status of the people. One of the policy objectives is to promote and sustain private public partnership in the delivery of health services. Policy strategies include poverty reduction, adoption of complementary health care financing options, which are sustainable involving PPP, coalition and multisectoral collaboration, involvement and representation of stakeholders and community health service delivery and establishing PPP in health provision.

Since at present private practitioners are regarded as partners in the provision of oral health care services rather than competitors, the role of the government is to provide a conducive environment for establishing private practice. With the introduction of private practice quality assurance of services provided should be given priority. In the Policy guidelines for oral health care (11) emphasis is put on the coverage of the rural population with basic oral health care services. Increasing access to oral health care to the rural population $(85 \%)$ can be attained through Public-Private Partnerships in Health by contracting private dentists to provide oral health care services in public health facilities.

\section{Applicable Policies and Procedures for contracting}

When private dentists contract with HF's to provide services to health facility patients within their own dental clinics, their clinics may be considered to be either satellites of the HF or off-site locations for contracted services. It may be preferable to characterize the dental clinic as an off-site location for services, rather than a satellite location, under terms of the contract. If a dental clinic is established as a satellite of the health facility, rather than simply as an off-site service location, it would 
need to comply with a range of facility requirements (12).

Both dentists and health facilities are required to provide all services in accordance with applicable laws, regulations, and policies and generally accepted principle of professional conduct. Health facilities are required to ensure that their in-house and contracted health care providers meet professional requirements including appropriate state licensure, certifications and registrations and maintain appropriate insurance coverage. Contracting dentists will need to provide health facilities with required credentialing information, including evidence of professional insurance coverage, so that the health facility can, in turn, meet its regulatory obligations.

When contracting to provide services to health facility patients, dentists and health facilities may elect to contract based on the specific services provided to the HF patient using a negotiated fee schedule; number of patients to be seen; number of visits available to HF patients; number of sessions (hours or days) to be committed to HF patients; or any other mutually agreeable basis. In all cases, the dentist and the health facility negotiate payment rates for an agreed-upon range of services. In this case services are provided to individual health facility patients without regard to the patient's ability to pay or pay source.

When contracting by service, patient, visit, or session, the dentist and the health facility negotiate a fixed payment for each such contracting unit. For example, contracting by patient obligates the dentist to provide a specified range of services for a specific number of patients over a designated period of time for a fixed charge per patient. Contracting by visit establishes a fixed payment rate for a visit regardless of the services provided or length of visit. Contracting by session obligates the parties to a specified number of clinical sessions which may be defined, for example, as a specified half-day each week or each month with half-day defined by agreed-upon hours. Regardless of approach, both the dentist and health facility should seek to determine anticipated costs and income when deciding on a payment methodology in order to limit financial risk to both parties. Ideally, the HF will limit its financial risk while assuring the dentist a predictable income for care of HF patients.

Specific services to be provided by the contracted dentist also are negotiable by the parties. As the HF agent, the dentist is not obligated to provide dental services that are not included in the agreement. HF's vary widely in the range of dental services they provide. Regardless of the range of services negotiated between the dentist and the health facility, HF's are required to ensure all services defined by their scope to all patients of the health facility, regardless of individual patients' ability to pay and, therefore, the health facility's ability to recoup costs of contracted care. Health facilities may elect to provide services beyond those required by law or included in their scope. For example, facilities that have not expanded their scope to include restorative dental services may nonetheless elect to provide these services.

\section{Accountability}

Patient protections that are typically afforded by dentists to all patients served in their clinics extend to health facility patients. Similarly protections afforded by health facilities to patients seen in their sites extend to health facility patients served at the private dental clinic as well. These protections include non-discrimination policies and practices, proper sterilization techniques, appropriate radiation safety procedures and other qualityassurance standards. Since the contracting private dentist acts as an agent for the health facility, the dentist is required to provide services to health facility patients in a manner that is consistent with applicable health facility policies and procedures. Both parties should carefully review their established policies and procedures including, but not limited to clinical protocols and guidelines, quality assurance standards and practices, standards of conduct and productivity standards to ensure or establish common expectations. Patient grievance procedures vary somewhat between private dentistry and HF practice. Dentists typically address patient grievances directly and seek to satisfy patients' concerns within their offices. A patient who remains unsatisfied may seek additional satisfaction, adjudication, or remedy from the state's dental licensing board, or through the courts. In contrast, HFs are required to establish formal patient grievance procedures. HF patients served by private dentists may access these procedures to address any grievance they may have. Dentists should become familiar with pertinent health facility policies and procedures before contracting with health facilities.

Good practice requires that both parties remain accountable to each other and address each other's needs in an ongoing way. Accordingly, good communication between the parties is essential to ensure satisfaction and programme accountability. Additionally, Government regulation and policy mandate that HF's be accountable for oversight of all contracted services provided to health centre patients. For example, it is important that dentists provide centers with information regarding progress in meeting the contracted goal, whether that be a specified number of patients, availability of care for specified sessions or visits, or numbers and types of services provided. Further, because payments made by health facilities to contracted providers must be reasonable as they relate to services provided, it is important that dentists provide a sufficient money value of services to substantiate the contracted 
payment amount. Under typical contracting arrangements, the health facility guarantees the dentist timely payment and the dentist agrees to provide health facility with necessary service delivery and financial reports reflecting his or her care of health facility patients.

Health facilities may need to access records maintained by the dentist that pertain to services provided to health facility patients in order to meet their performance, quality assurance, and general monitoring and oversight requirements. Since the contract is expected to be typically paid with government funds, the dentist also may be asked by appropriate governmental funding agencies to provide access to patient's records.

\section{Conclusion}

Public Private Partneships provides opportunity for looking at health in a comprehensive way. It builds on the strengths of each organisation, allowing them to pool resources, avoid duplication and minimize impact. PPPH also encourege the involvement of communities in their health care and focus attention on neglected health problems, attracts new resources and use them efficiently, fill service gaps and increase access to services for underserved groups. Since private practitioners are regarded as partners in the provision of health care services rather than competitors, increasing access to oral health care in the rural areas can be attained through contracting private dentists to provide oral health care services in Public Health Facilities in these areas. When contracting to provide services to health facility patients, dentists and health facility may elect to contract based on the specific services provided to the HF patient using a negotiated fee schedule; number of patients to be seen; number of visits available to HF patients; number of sessions (hours or days) to be committed to HF patients; or any other mutually agreeable basis. Since the contracting private dentist acts as agent for the health facility, the dentist is required to provide services to health facility patients in a manner that is consistent with applicable health facility policies and procedures. If the Government could provide a conducive environment for establishing private dental practice, the coverage of the rural population with basic oral health care services can be attained as outlined in the National Plan for Oral Health (11) through contracting with private dentists to provide oral health care services in the HFs located in these areas..

\section{References}

1. World Health Organization. Oral health care systems. An International Collaborative Study 1985, Geneva, Switzerland

2. World Health Organization. Comparing Oral Health Care Systems. A second International Collaborative Study.1987 Geneva, Switzerland.

3. Hoffman AW. History of Dentistry 1981 Chicago IL. Quintessence.

4. Dunning JM. The Social Sciences. In Dunning JM ed. Principles of dental public health, $4^{\text {th }} \mathrm{Ed}$ Cambridge, MA, Harvard University Press 1986.

5. Harris RR. Dental Science in a new age. A history of the National Institute of Dental Research. Rockville, MD, Montrose Press 1989.

6. Gallagher EB. Modernization and medical care. In: Conrad P, Gallagher EB eds. Health care in developing countries. Sociological perspective. Philadelphia PA. Temple University Press 1993

7. Beetstra S, Derksen D, Marguerite Ro, Powell W, Donald EF, MD, and Kaufman A. A Health Commons Approach to Oral Health for LowIncome Populations in a Rural State 2002:92! 12-13

8. Ministry of Health. Health Sector Strategic Plan (HSSP) 2003.

9 Kirunga C Forni C, Bataringaya J. PublicPrivate Partnership in Health (PPPH): Increasing Private Health Sector participation in all aspects of the National Health Programme Ministry of Health, Uganda 2006

10. Ministry of Health. National Health Policy 2002.

11. Ministry of Health. Policy Guidelines for Oral Health Care in Tanzania 2002

12 Burton L. Edelstein D. Increasing Access to Dental Care through Public/Private Partnerships: Contracting between Private Dentists and Federally qualified Health Centres. A Handbook. 2003: 12-16

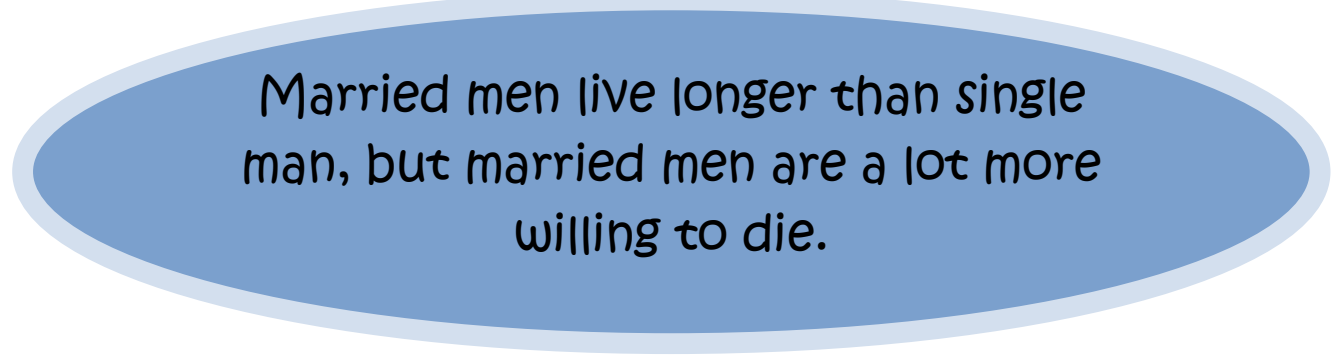

